\title{
Hydroxyurea-induced superinfected ulcerations: Two case reports and review of the literature
}

\author{
GABRIELA MARIANA IANCU ${ }^{1,2}$, ANCA OCNEANU ${ }^{3}$ and MARIA ROTARU ${ }^{1,2}$ \\ ${ }^{1}$ Dermatology Department, Faculty of Medicine, University Lucian Blaga of Sibiu, 550169 Sibiu; ${ }^{2}$ Clinic of Dermatology, \\ County Emergency Hospital of Sibiu, 550245 Sibiu; ${ }^{3}$ Dermatology Office, Grivita Medical Center, 810521 Braila, Romania
}

Received July 10, 2020; Accepted August 10, 2020

DOI: 10.3892/etm.2020.9321

\begin{abstract}
The chronic use of hydroxyurea (HU) in some oncologic and non-oncologic diseases (psoriasis, sickle cell anemia) can be accompanied by side effects, both systemic and mucocutaneous. The most severe adverse events known in HU therapy are leg ulcers and cutaneous carcinomas. At skin level may also appear: xerosis, persistent pruritus, skin color changes (erythema, hyperpigmentation), cutaneous atrophy. Likewise, oral ulcerations and stomatitis may occur at mucosal level. Hair damage can be expressed through alopecia and nail damage through melanonychia and oncycholysis. First case, a 63-year-old woman with severe psoriasis vulgaris and chronic granulocytic leukemia, with 5 years of HU therapy, was admitted to hospital for submammary and palmoplantar ulcers, superinfected with methicillin-resistant Staphylococcus aureus and Proteus mirabilis. Clinical exam showed that the patient had also cutaneous atrophy, marked palmoplantar xerosis and melanonychia. The second case, a 72-year-old woman with primary thrombocytemia, treated with HU for 3 years, presented with necrotic leg ulcers that were superinfected with Pseudomonas aeruginosa, Enterobacter and E. Coli. The patient associates cellulitis, microbial eczema and xeroderma. In both cases, after HU discontinuation, systemic antibiotics, topical epithelizing agents and emollients, the ulcers had a slow favorable evolution. In our cases, the ulcers appeared after 5 , respectively 3 years of HU therapy. It is stressed that in the first case, which had associated psoriasis, after 1 year of $1 \mathrm{~g}$ of HU/day, the psoriatic lesions completely disappeared. The severe progression of the ulcers was also favored by the superinfection of the ulcers with 2, respectively, 3 identified germs for which appropriate systemic antibiotics was required.
\end{abstract}

Correspondence to: Dr Gabriela Mariana Iancu, Dermatology Department, Faculty of Medicine, University Lucian Blaga of Sibiu, 2A Lucian Blaga Street, 550169 Sibiu, Romania

E-mail:mgabiancu@yahoo.com

Key words: hydroxyurea, side effects, cutaneous, leg ulcers, superinfected

\section{Introduction}

Hydroxyurea (HU) is a urea hydroxylated derivate used for nearly 60 years as a chemotherapeutic agent, effective in the treatment of certain neoplasias: Hematological (chronic myeloid leukemia resistant to treatment), inoperable brain, kidney, breast, cervical and cutaneous cancers (squamous cell carcinoma, malignant melanoma). Besides, HU can also be used in the treatment of non-neoplastic diseases (sickle cell anemia, psoriasis, HIV infection) (1).

In terms of pharmacokinetics, HU is intestinally absorbed, metabolized by the liver and urinary excreted (1). After absorption, it is transformed into a nitroxide free radical and transported by diffusion to the cells presenting the active site of the M2 protein subunit of the ribonucleotide reductase, which is inactivated. DNA synthesis is selectively inactivated by apoptosis in the $\mathrm{S}$ phase of the cellular cycle. HU inhibits DNA repair, having synergistic action with ultraviolet radiation or with alkylating agents. HU also shows radiosensitization activity by maintaining the cells in radiation-sensitive G1 phase and interfering with DNA repair (2).

The anti-neoplastic mechanism of action is not fully elucidated. Some studies indicate that HU interferes with DNA synthesis without interfering with RNA or protein synthesis. Although it has numerous sites of action, it most likely inhibits the incorporation of thymidine into DNA by its direct action and also the free tyrosyl radical residue (the catalytic center of ribonucleotide diphosphate reductase, the enzyme which catalyzes the transformation of ribonucleotides into deoxyribonucleotides). $\mathrm{HU}$ is an inhibitor of the $\mathrm{S}$ phase of the cellular cycle, determining cell sequestration in G1-S phase, decreasing cell progression rate in $\mathrm{S}$ phase, and/or determining accumulation of cells in $\mathrm{S}$ phase as a result of DNA synthesis inhibition (2).

Studies conducted on animals have shown that the cytotoxic effects of HU are limited to the tissues with high cellular proliferation rates and the effects are noticeable in cells with active synthesis of DNA (3). Thus, the teratogenic effects of the chemotherapeutic agent are explained.

HU also presents a number of systemic side effects, of which the most important are hematological (pancytopenia or cytopenia on certain hematological lines), gastrointestinal (dyspeptic syndromes), neurological (headache, vertigo, hallucinations, seizures), renal (hyperuricemia, renal lithiasis). 
Skin reactions occur in $10-30 \%$ of patients chronically treated with HU. The most common are pruritus, facial and acral erythema and xeroderma, skin hyperpigmentation, leg ulcers and stomatitis. Exacerbation of post-radiotherapy erythema (in case of associated radiotherapy), affection of the nails (melanonychia, onycholysis, blue lunula), skin atrophy and alopecia may also occur.

The increasing number of new therapies used to stabilize oncological diseases determines the increment of the common mucocutaneous side effects in dermatological practice. The greatest use of HU in the hospital is in hemato-oncology clinic. Most frequently we have controllable side effects, only with compensatory dermatological therapy. Rarely, in selected cases, the severity of the skin manifestations requires the discontinuation of HU therapy. We present two cases with severe skin adverse events (leg, submammary and palmoplantar ulcers) in patients undergoing chronic HU treatment for chronic granulocytic leukemia (CGL), respectively primary thrombocythemia, which required the discontinuation of $\mathrm{HU}$ therapy and the modification of oncological therapy.

\section{Case reports}

Case 1. A 63-year-old female patient with type 2 diabetes, chronic venous insufficiency (venous ulcer epithelized in 2016), severe psoriasis vulgaris since 2001 with many episodes of erythroderma, treated with Methotrexate then with Acitretin, was diagnosed with CGL (2012) for which HU treatment was followed for 5 years. For CGL, the patient initially followed a treatment with Imatinib which was not tolerated due to severe thrombocytopenia. After 2 months she presented with multiple palmoplantar and submammary, painful, bleeding ulcers, which progressively aggravated with depth extensions (Fig. 1). Of note is the complete remission of psoriasis lesions over the last 4 years without specific therapy for psoriatic disease. On admission the patient presented also skin atrophy, generalized xerosis and melanonychia.

Laboratory investigations showed moderate anemia (Hb $9.3 \mathrm{~g} / \mathrm{dl}$, Ht 27.7\%), inflammatory syndrome (ESR $63 \mathrm{~mm} / \mathrm{h}$ ), superinfection of ulcers with MRSA Staphylococcus aureus and Proteus mirabilis and positive urine cultures with E. Coli.

In order to control the adverse events of the skin, the hematologist recommended a temporary discontinuation of HU. Superinfection of ulcers was treated with Cefuroxime $3 \mathrm{~g} /$ day. Topical treatment of ulcers was performed with disinfection and application of epithelizing agents. The xerosis was controlled with emollients. In evolution, the ulcers slowly epithelialized and the skin texture improved (Fig. 2).

Case 2. A 72-year-old female, diagnosed in 2014 with primary thrombocytemia (treated with Thromboreductin $2.5 \mathrm{mg}$ /day and HU $500 \mathrm{mg} /$ day for 3 years) was admitted for giant, necrotic leg ulcers, extremely painful, appeared in the previous 2 months. A well-defined, pruriginous, erythematous plaques covered with squamo-crusts, edema (Fig. 3A) and marked skin xerosis were present around the ulcer.

On admission we had an underweight patient (body mass index $18.3 \mathrm{~kg} / \mathrm{m}^{2}$ ), with a kyphoscoliotic thorax, with abolished vesicular murmur in the right lung base and with

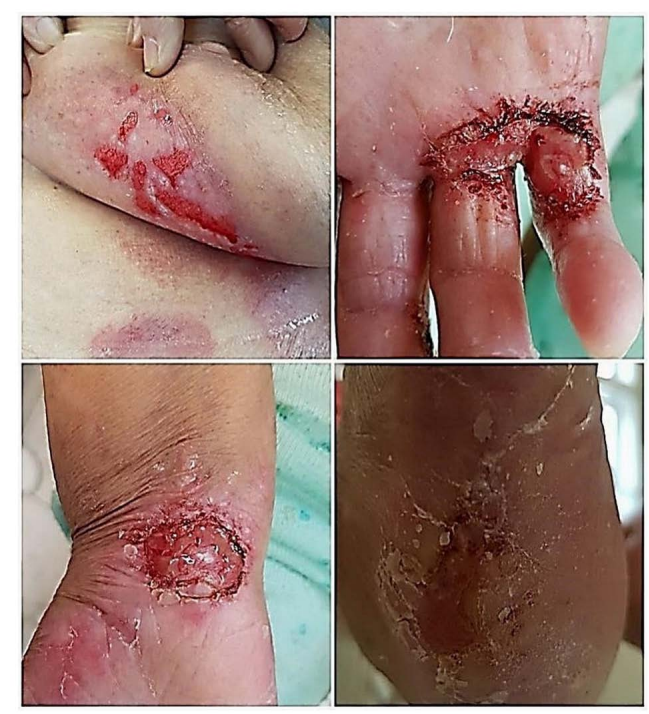

Figure 1. Submammary, palmoplantar and radiocarpian ulcers on admission.

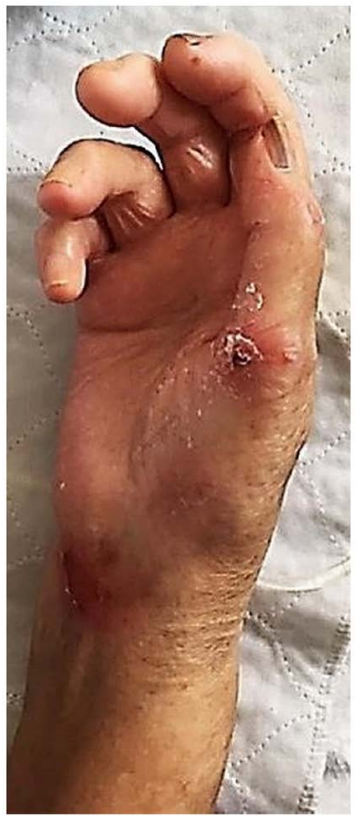

Figure 2. At 3-month follow up (epithelization of ulcers, improving xerosis, persistence of melanonychia).

slightly elevated blood pressure $(160 / 80 \mathrm{mmHg})$. The laboratory investigations showed a hypochromic macrocytic anemia (Hb $9.6 \mathrm{~g} / \mathrm{dl}$, Ht 30, 11\%), leukocytosis with neutrophilia (L 24.440/mm $\mathrm{mm}^{3}$, Ne 89.6\%), mild thrombocytosis ( $\mathrm{Tr}$ $484000 / \mathrm{mm}^{3}$ ). The bacteriological exam of the ulcer identified a triple association of germs [Pseudomonas aeruginosa, Enterobacter ESBL (+) and E. Coli ESBL (+)]. Chest radiography revealed a right basal pneumonia with pleural effusion.

Due to the triple association of germs and pulmonary congestive process, we decided to start the treatment with Cefuroxime $3 \mathrm{~g} /$ day, 10 days, with probiotics, antimycotics, analgesics and diuretics. The therapeutic approach regarding the ulcers was a classical one with disinfectants and silver nitrate epithelizing creams. Hematologist decided to discontinue the HU administration and increasing the dose of 

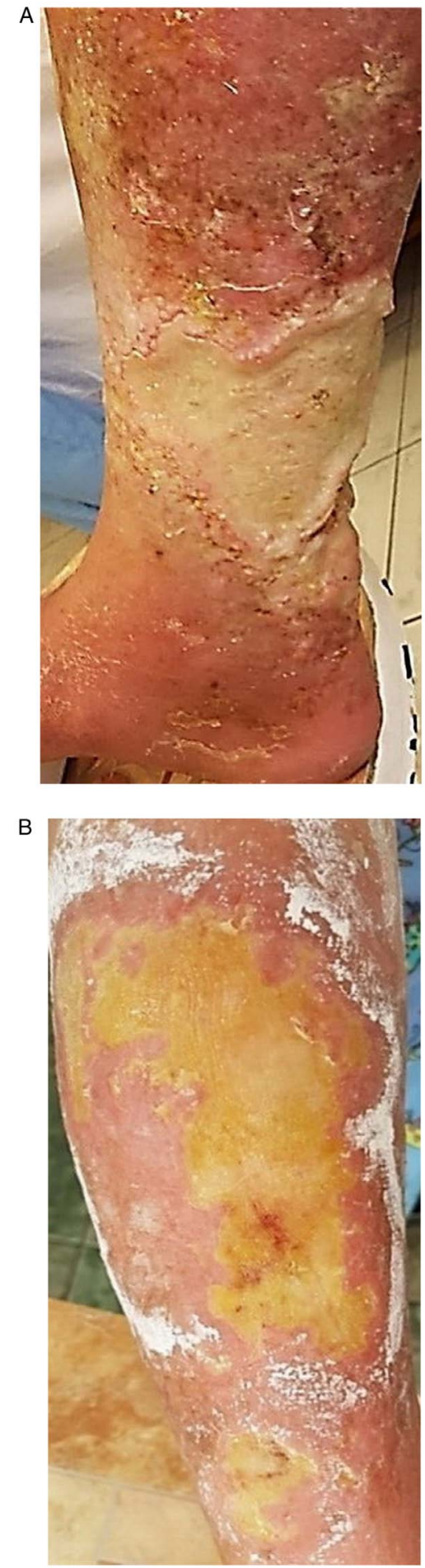

Figure 3. (A) Giant, superinfected ulcers with secondary cellulitis on admission. (B) A 1-month ulcer follow-up (reduction in size, reduction of fibrino-purulent exudate and cellulite healing).

Thromboreductin, which allowed a slow epithelialization of ulcers (Fig. 3B).

Both patients or their family gave written inform consent for publication.

\section{Discussion}

Leg ulcers appear in approximately $9 \%$ of patients who receive high doses of $\mathrm{HU}$, administered chronically. Chaine et al reported a higher incidence of HU induced leg ulcers (29\%) (4). The ulcers are more frequent in the malleolus region and more painful for women who have been treated with HU for at least 1 year (65\%). The skin around the ulcer usually presents atrophies blanche (histopathological-epidermal atrophy, dermal fibrosis with scar tissue but without vascular damage) (5).

Leg ulcers occur by reducing tissue viability when the chemotherapeutic agent disrupts the $\mathrm{S}$ phase of the cellular cycle. HU inhibits DNA synthesis, damaging the basal keratinocytes and disruption of collagen production (5). From the pathophysiological point of view, leg ulcers induced by chronic HU therapy appear by lowering the blood flow at microcirculation level. The anoxia secondary to chemotherapy induced macrocytosis and the formation of microthrombi. Healing occurs within 2-9 months after stopping the HU treatment. Some authors consider it necessary to stop the treatment only in cases of refractory, progressive leg ulcers (6). Other methods for ulcer treatment are textile biomaterials impregnated with active principles (phlebotonics, heparin-drugs, capillarotrophic drugs) (7).

In the first case, after 5 years, the HU therapy induced the occurrence of ulcers with particular submammary and acral disposition and marked cutaneous xerosis and melanonychia. In this case, the HU therapy was beneficial in psoriasis evolution with the disappearance of psoriatic lesions after 1 year of treatment. Even if the patient had experienced challenging, hardly manageable episodes of psoriasis erythroderma in the past, before taking $\mathrm{HU}$, over the last 4 years she has not had any psoriasis lesions (she reached PASI 0-absolut psoriasis area and severity index). In psoriasis the interconnections between the immune system, the nervous system (expressed by stress that triggers the first or the new episodes of skin lesions) and the skin are very complex (8). To control this disease, a variety of treatment schemes were tested, including HU. The therapeutic effect of $\mathrm{HU}$ in psoriasis is based on the antimitotic effect of HU (reduces DNA replication in the basal layer).

Oakley (9) showed that in half of the patients with psoriasis, resistant to some conventional therapies (Methotrexate, Acitretin or phototherapy), HU therapy determines a favorable response. The first results appear after 2 weeks of treatment and the response is within 8 weeks. In our case, PASI 0 was reached after 1 year of $\mathrm{HU}$ treatment. It has also been found that doses of $500 \mathrm{mg}$ up to $1 \mathrm{~g} /$ day of HU have therapeutic effects in selected cases of psoriasis. In our case, $1 \mathrm{~g} /$ day of HU was the therapeutic dose for both psoriasis and CGL but unfortunately with the occurrence of adverse events (ulcerations with particular sites, xerosis, melanonychia) after 5 years of treatment. In the second case, skin reactions secondary to HU treatment appeared after 3 years of therapy and were among the most commonly encountered: Leg ulcers and xeroderma.

The mucocutaneous and nail hyperpigmentation induced by chronic HU therapy is due to genetic predisposition, photosensitivity with focal stimulation of melanocytes and cytotoxic effect on the nail bed and matrix. A plausible hypothesis is that the activation of melanocytes inducted by drugs increased the production of melanin (6). Hoff et al (10) observed that in patients chronically treated with a chemotherapeutic agent, skin hyperpigmentation is more common, without nail damage, and the isolated presence of melanonychia is rarely encountered. The pigmentation changes induced by HU occur 
especially in adult patients with dark phototype and sickle cell anemia (11).

The nail changes that may occur are longitudinal nail bands with varying pigmentation intensity, usually on a few nails, especially those on the hand. In specialized literature only 4 cases were reported in which nail damage was observed in all 20 nails in the same patient (12). Other changes that may sometimes occur simultaneously are transversal bands and diffuse pigmentation. Also, melanonychia appeared in $4 \%$ of the patients who underwent chronic HU treatment over a long period of time (13). In our cases, nail hyperpigmentation remitted after few months of HU therapy discontinuation.

Rarely, in the course of chronic therapy with HU, dermatomyositis-like lesions, skin carcinomas (squamous cell carcinomas, rarely basal cell carcinoma) (3\%) (4) or precancerous lesions may occur. Skin carcinomas occur due to the mutagenic effect of the chemotherapeutic agent that determines the inhibition of DNA repair, damaged by external factors (especially ultraviolet radiation). The appearance of skin carcinomas requires a surgical treatment, general measures with clothing and cream photo-protection with high sunscreen protection factor. In our case, the decision to discontinue the HU therapy was taken by the hematologist, balancing the benefits and risks of this therapy (14).

In conclusion, in over 1 year of HU treatments, the mucocutaneous adverse effects are frequent. The mechanism by which these side effects occur is not fully known. In the presented cases, the mucocutaneous manifestations secondary to chronic HU therapy were leg ulcers with particular localizations (submammar, palmoplantar), xeroderma, skin atrophy, melanonychia. In the first case, the beneficial effect of HU on the evolution of psoriatic disease should be mentioned, obtaining PASI absolute after 1 year of treatment. In the cases presented by us, leg ulcers and those with particular localization (palmoplantar and submammary) occurred after 5 years, respectively, 3 years of HU therapy initiation. The healing process required HU discontinuation, association of systemic antibiotics for superinfected ulcers, and topical epithelizing treatment.

\section{Acknowledgements}

Not applicable.

\section{Funding}

No funding was received.

\section{Availability of data and materials}

The datasets used and/or analyzed during the current study are available from the corresponding author on reasonable request.

\section{Authors' contributions}

GMI and MR contributed in the conception and design of the study, analysis and interpretation of patient data, manuscript drafting and critical revision of the manuscript for important intellectual content. AO contributed in the data acquisition, analysis and interpretation of patient data, manuscript drafting and design of the study. All authors read and approved the final manuscript.

\section{Ethics approval and consent to participate}

Not applicable.

\section{Patient consent for publication}

The patients or their family gave written inform consent for publication.

\section{Competing interests}

The authors declare that they have no competing interests and they have no financial relationships to disclose.

\section{References}

1. Gwilt PR and Tracewell WG: Pharmacokinetics and pharmacodynamics of hydroxyurea. Clin Pharmacokinet 34: 347-358, 1998.

2. Yarbro JW: Mechanism of action of hydroxyurea. Semin Oncol 19 (3 Suppl 9): S1-S10, 1992.

3. National Library of Medicine (US), National Center for Biotechnology Information: PubChem Compound Summary for CID 3657, Hydroxyurea. https://pubchem.ncbi.nlm.nih.gov/compound/ Hydroxyurea. Accessed August 4, 2020.

4. Chaine B, Neonato MG, Girot R and Aractingi S: Cutaneous adverse reactions to hydroxyurea in patients with sickle cell disease. Arch Dermatol 137: 467-470, 2001.

5. Dissemond $\mathbf{J}$ and Körber A: Hydroxyurea-induced ulcers on the leg. CMAJ 180: 1132, 2009.

6. França ER, Teixeira MA, Matias Kde F, Antunes DE, Braz Rde A and Silva CE: Cutaneous effects after prolonged use of hydroxyurea in polycythemia Vera. An Bras Dermatol 86: 751-754, 2011.

7. Branisteanu DE, Nichifor M, Dorobat CM, Branisteanu DC, Petrariu FD, Molodoi AD, Radu DC and Boda D: Use of textile biomaterials for the topic treatment of chronic venous disease. Rom Biotechnol Lett 20: 10618-10625, 2015.

8. Grigore O, Mihailescu AI, Solomon I, Boda D and Caruntu C: Role of stress in modulation of skin neurogenic inflammation. Exp Ther Med 17: 997-1003, 2019.

9. Oakley A: Hydroxyurea. DermNet NZ, New Zealand, 2001. https://www.dermnetnz.org/topics/hydroxyurea. Accessed June 23, 2019.

10. Hoff NP, Akanay-Diesel S, Pippirs U, Schulte KW and Hanneken S: Cutaneous side effects of hydroxyurea treatment for polycythemia Vera. Hautarzt 60: 783-787, 2009 (In German).

11. Soutou B and Aractingi S: Myeloproliferative disorder therapy: Assessment and management of adverse events - a dermatologist's perspective. Hematol Oncol 27 (Suppl 1): S11-S13, 2009.

12. Gropper CA, Don PC and Sadjadi MM: Nail and skin hyperpigmentation associated with hydroxyurea therapy for polycythemia Vera. Int J Dermatol 32: 731-733, 1993.

13. Neynaber S, Wolff H, Plewig G and Wienecke R: Longitudinal melanonychia induced by hydroxyurea therapy. J Dtsch Dermatol Ges 2: 588-5891, 2004 (In German).

14. Wiechert A, Reinhard G, Tüting T, Uerlich M, Bieber T and Wenzel J: Multiple skin cancers in a patient treated with hydroxyurea. Hautarzt 60: 651-652, 654, 2009 (In German).

This work is licensed under a Creative Commons Attribution-NonCommercial-NoDerivatives 4.0 International (CC BY-NC-ND 4.0) License. 